\title{
Compact Planar Microwave Blocking Filter
}

\author{
Kongpop U-yen ${ }^{\# 1}$ and Edward J. Wollack ${ }^{\# 2}$ \\ "NASA Goddard Space Flight Center \\ Greenbelt, Maryland, USA \\ 'kongpop.u-yen-19nasa.gov \\ 'edward.j.wollack@nasa.gov
}

\begin{abstract}
This paper proposes a technique to design a compact planar broadband microwave blocking filter. The filter is constructed from multiple sections of bandstop filters with means to control radiation loss. As a result, the rilter has small physical size and provides multi-decade of suppression frequency bandwidth. The total length is less than half-wavelength long at the 3-dB corner frequency of $1.45 \mathrm{GHz}$. The experimental results show that the microstrip microwave blocking filter can provide an attenuation of more than $70 \mathrm{~dB}$ from $7 \mathrm{GHz}$ to $39.5 \mathrm{GHz}$ with the total DC capacitance of less than $23 \mathrm{pF}$.
\end{abstract}

\section{INTRODUCTION}

Microwave themal blocking filters find use in cryogenic electronics systems containing such low-noise devices as Josephson tunnel junctions, coulomb blockade devices, and bolometric detectors. The thermal blocking filter is used to realize the necessary isolation between the cooled elements of the device and room temperature readout and bias electronics. The use of this component can prevent the degradation of the detector performance by the Johnson noise emitted from warmer elements of the electronics required by the sensor system.

Although providing a low-noise DC bias line is in principle very simple using large value shunt capacitor [1], realizing this function with a broadband readout capability can be challenging. Several techniques have been used to obtain this capability.

A dissipative solution is to use a resistor loaded filter [2][7], where microwave power is absorbed along the structure to provide very broadband attenuation. However, to provide sufficient attenuation at high frequency, the filter requires a long line which makes it highly capacitive and limits the operating bandwidth of the signal. In addition this technique requires the use of lossy or loaded dielectric materials, which are not compatible with thin film fabrication processes.

The non-dissipative techniques can also be effective at blocking the thermal noise power, however, design must adequately addressed spurious transmission resonances and sensitivity to impedance matching. Multiple low-pass filters can be combined in series to produce broadband rejection responses [8-9]. The cascade filter in [8] can produce wide stopband bandwidth using various sizes of hairpin resonators. However, the overall filter requires a large metal area which contributes to high equivalent DC shunt capacitance. Although the filter with slots on the ground plane [9] can provide broadband attenuation with miniature size, its attenuation level can be limited at high frequency due to the strong radiation from slot line structures and inadequate field confinement.

In this paper, we propose a compact filter design technique using a cascaded bandstop filter that produces very broadband microwave blocking capability. In addition, techniques to reduce the filter's equivalent DC shunt capacitance and control radiation loss are discussed. Finally a hardware prototype is developed to validate the filter design.

\section{FILter DESIGN Procedures}

Since this filter will be used at the input of the analog voltage readout with bandwidth of less than $300 \mathrm{MHz}$, it is necessary for the filter to have a low equivalent shunt capacitance and a low series resistance. In addition, the low frequency analogue input impedance is higher than $50 \mathrm{Ohm}$ which relaxes the impedance matching requirement at the filter's input ports.

The concept design of this microwave blocking filter is to combine many compact bandstop filters. These bandstop filters are scaled to suppress various frequency bands such that the microwave blocking filter provides very low radiation leakage and has a viable means of achieving targeted total filter shunt capacitance of $<20 \mathrm{pF}$. For this reasons, stepped impedance coupled line filters [10] are selected as a unit element of the proposed microwave blocking filter. Its circuit model (shown in Fig 1(a)) consists of two sections: the coupled line section with characteristic impedance $\left(Z_{c}\right)$ and a low impedance open-ended line with characteristic impedance $\left(Z_{l}\right)$.

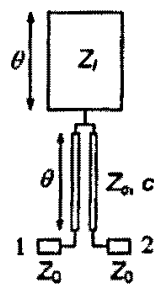

(a)

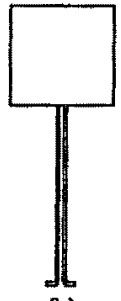

(b)

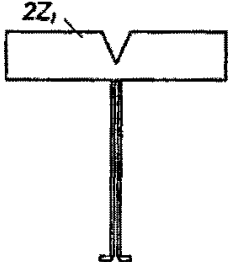

(c)

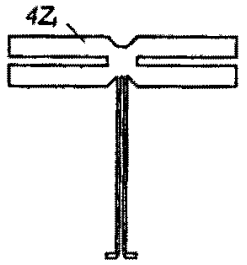

(d)
Fig. 1. Three different types of unit element of the proposed microwave blocking filter design: (a) circuit model and physical realization in microstrip lines with (b) conventional $Z_{l}$ termination, (c) the proposed split-end $Z_{i}$ termination and (d) quadruple-end $Z_{\text {, }}$ termination.

\section{A. Unit element design}

The unit element is based on the broadband bandstop filter described in [10]. It offers compact realization of the low-pass 
filter by designing the filter at a much higher frequency than the filter's $3 \mathrm{~dB}$ comer frequency. In addition, each unit element is adjusted with same electrical length to ideally generate three transmission zeros frequencies $f_{0}, f_{1}$ and $f_{2}$ in the stop-band, thus maximizing the bandwidth, as shown in Fig. 2.

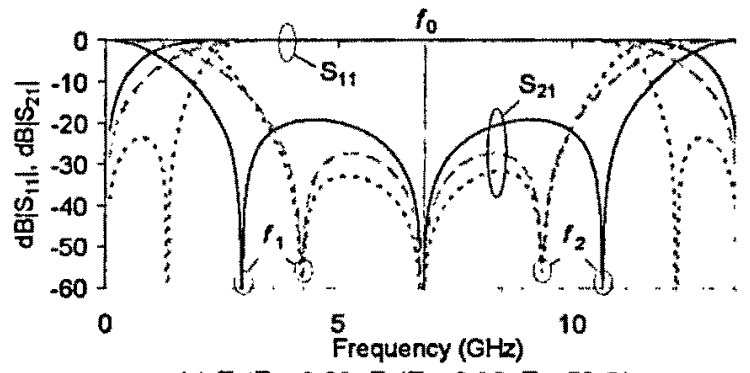

(a) $Z_{i} / Z_{c}=0.22, Z_{d} Z_{c}=0.86, Z_{0}=53 \mathrm{Ohm}$

(b) $Z_{1} / Z_{\mathrm{c}}=0.11, Z_{\mathrm{d}} Z_{\mathrm{c}}=0.86, Z_{0}=53 \mathrm{Ohm}$

.... (c) $Z_{d} / Z_{c}=0.11, Z_{d} Z_{c}=0.43, Z_{0}=26 \mathrm{Ohm}$

Fig. 2. The frequency response of three unit elements designed at $f_{0}=6.8 \mathrm{GHz}$ by varying $Z_{0} / Z_{\tau}$ from 0.86 to 0.43 and by varying $Z_{s} / Z_{c}$ from 0.11 to 0.22 . $c=0.26$.

This condition occurs when the electrical lengths $(\theta)$ in Fig 1 of both $Z_{c}$ and $Z_{l}$ sections equal to quarter-wavelength long at the center frequency $f_{0}, f_{1}$ and $f_{2}$ are analytically determined as follows

$$
\frac{f_{1}}{f_{0}}=\frac{2}{\pi} \tan ^{-1} \sqrt{\frac{2 Z_{1}}{Z_{c, s}-Z_{c, o}\left(1+2 Z_{l} / Z_{c, s}\right)}}
$$

and

$$
\frac{f_{2}}{f_{0}}=2-\frac{f_{1}}{f_{0}}
$$

Where $Z_{c, e}$ and $Z_{c, 0}$ are even- and odd-mode characteristic impedances of the coupled line $Z_{c}$. The coupling coefficient $c$ of $Z_{c}$ is defined as $c=\left(Z_{c, e}-Z_{c, o}\right)\left(Z_{c, e}+Z_{c, o}\right)$.

The filter length is reduced by using a small $f_{1} / f_{0}$ ratio. From (1), this can be achieved by adjusting $c_{\mathrm{z}}$ and $Z_{1}$. From (1) and (2), increasing $Z_{0} / Z_{c}$ ratio does not affect $f_{1}$ and $f_{3}$, however it increases the filter's stopband attenuation and passband return loss level as shown in Fig. 2(c).

To minimize the total capacitance, we set $Z_{c}$ and $c$ at the highest allowable value by the fabrication process. $Z_{0}$ is also set to a high value to minimize the line capacitance that will be used to connect among unit elements. Due to these restrictions, the filter will be operating in the condition shown in Fig 2(a).

\section{B. Filter pass-band design}

The passband for the microwave blocking filter is defined by $f_{1}$ of the unit element. Since this band stop filter is designed at low frequency below $2 \mathrm{GHz}$, it can have long transmission line physical length. The filter size can be reduced significantly by designing the filter at $f_{0}$ that is much greater than $f_{1}$. From (1), we adjust $Z_{l}$ to the lowest value of $7 \mathrm{Ohm}$. However, this requires wide transmission line in the $Z_{l}$ section (as shown in Fig 1(b)), which increases the filter's equivalent shunt capacitance. To reduce this capacitor without affecting the filter passband response, we proposed to split $Z_{l}$ into two and four sections. Each section has an impedance of $2 Z_{l}$ and $4 Z_{l}$, as shown in Fig. 1(c) and $1(\mathrm{~d})$, respectively. The physical length of $Z_{l}$ is shorter than that of $Z_{c}$ to compensate for the parasitic due to $Z_{\mathrm{I}} / Z_{\mathrm{c}}$ step discontinuity. Their passband frequency responses in Fig. 3(a) agree well with the ideal response from the theoretical model. However, they have strong deviation from the ideal response above $f_{1}$. This is due to the strong parasitic from step discontinuity between $Z_{l}$ and $Z_{c}$ that causes mismatch in odd- and even-mode propagation constants. In addition, the filter with $Z_{1}$ split into four sections generates spurious response between 8 and $9 \mathrm{GHz}$. This is due to unequal effective electrical length among four split sections.

Despite its strong out-of-band spurious responses, the shunt capacitance in the $Z_{l}$ section is reduced from $4.85 \mathrm{pF}$ in Fig I(b) to $4.74 \mathrm{pF}$ and $4.31 \mathrm{pF}$ in Fig. 1(c) and 1 (d), respectively. The structure in Fig. 1(d) also produces lower radiation loss at low frequency (below $6 \mathrm{GHz}$ as shown in Fig. 3(b), but it produces higher radiation loss out of band. This loss can be further suppressed and controlled by enclosing the filter in an appropriate cavity as will be discussed in section $C$.

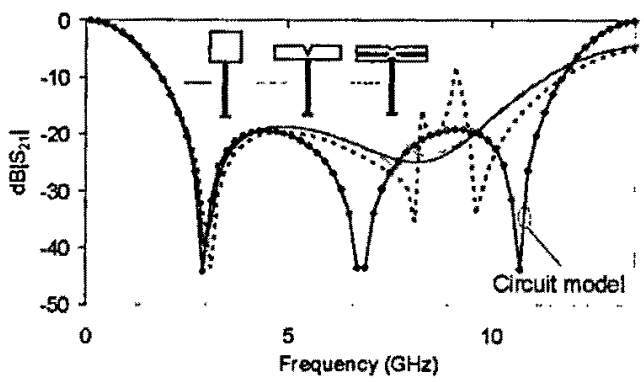

(a)

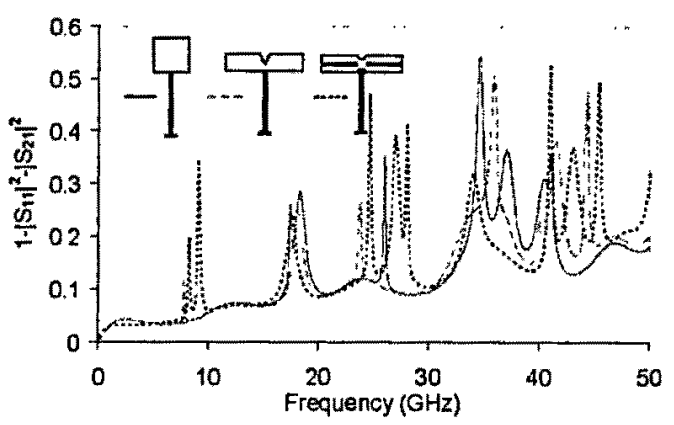

(b)

Fig. 3. Simulated frequency responses of $S_{21}$ and the total loss of the microstrip unit elements using three different types of $Z_{1}$ sections on 0.127 mm-thick Roger's Duroid 6010 substrate. Their frequency responses are compared with the ideal circuit responses with parameter values shown in Fig. 2(a)

C. Filter stop-band design 


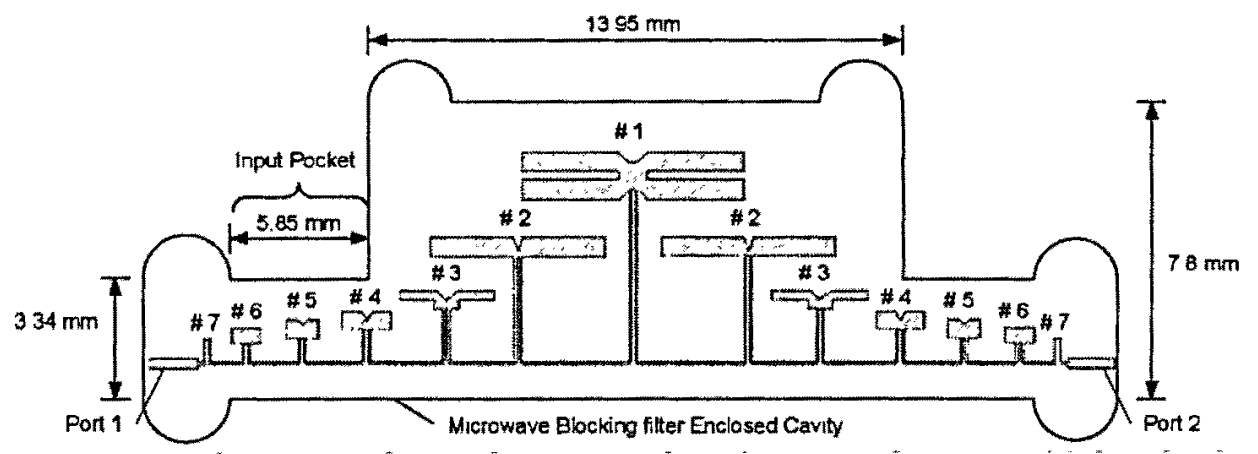

Fig. 4. The layout of the microstrip microwave blocking filter in an enclosed cavity.

Before the filter stopband can be designed, we must determine the upper frequency limits the filter can be used. These limits are maximum stopband attenuation and operating frequency, which are dependent on the actual implementation, i.e. size and type of dielectric, enclosed area and the transmission line fabrication resolution.

Microstrip transmission is chosen as a transmission medium since it has good electric field confinement and is simple to fabricate. An electrically thin dielectric substrate is used in the design to avoid surface wave propagation at the highest frequencies of interest [11]. Metal wall will be used to enclose the structure to prevent radiation from large structure to the filter's terminals from propagation unimpeded through the filter housing. The physical lifnits due to these factors are computed in terms of maximum operating frequency and summarized in Table I.

Table I the microw ave blocking upper frequency limits due to propagation mode in three different mediums.

\begin{tabular}{|l|l|l|l|}
\hline $\begin{array}{l}\text { Propagation } \\
\text { Medium }\end{array}$ & $\begin{array}{l}\text { Minimum } \\
\text { Design } \\
\text { Feature size }\end{array}$ & $\begin{array}{l}\text { Major } \\
\text { Frequency } \\
\text { Limitations }\end{array}$ & $\begin{array}{l}\text { Maximum } \\
\text { filter design } \\
\text { frequency }\end{array}$ \\
\hline $\begin{array}{l}\text { Microstrip } \\
\text { line on } \\
0.13 \mathrm{~mm} \text {-thick } \\
\text { substrate }\end{array}$ & $\begin{array}{l}0.18 \mathrm{~mm} \times \\
0.63 \mathrm{~mm}\end{array}$ & $\begin{array}{l}\text { line } \\
\text { width/length } \\
\text { ratio }\end{array}$ & $45.5 \mathrm{GHz}$ \\
\hline $\begin{array}{l}\text { Substrate with } \\
\varepsilon_{\mathrm{T}}=10.2\end{array}$ & $\begin{array}{l}0.127 \mathrm{~mm} \\
\text { thick }\end{array}$ & $\begin{array}{l}\text { Surface wave } \\
\text { TE0 cut-off } \\
\text { frequency [11] }\end{array}$ & $195 \mathrm{GHz}$ \\
\hline $\begin{array}{l}\text { Metal } \\
\text { enclosure }\end{array}$ & $\begin{array}{l}3.34 \mathrm{~mm} \times \\
2.54 \mathrm{~mm} \\
\text { cross- } \\
\text { sectional area }\end{array}$ & $\begin{array}{l}\text { Waveguide } \\
\text { Cavity } \\
\text { resonances } \\
\text { TE10 cut-off } \\
\text { frequency [12] }\end{array}$ & $45 \mathrm{GHz}$ \\
\hline
\end{tabular}

From Table $I$, the maximum frequency the filter can operate is limited by the microstrip physical dimensions. Therefore, the small metal enclosure is designed to suppress waveguide propagation mode above $45 \mathrm{GHz}$ and filter is constructed using seven different element sizes connected in series as shown in Fig. 4. The element described previously is reused with the electrical length scaled to operate at various frequency bands. Each of which is designed such that its stopband frequencies overlapped with the adjacent one. The minimum feature used in this design is a quarter-wavelength stub in section $\$ 7$ and it is limited to fabrication resolution. It is used to suppress the spurious responses at $45.5 \mathrm{GHz}$. The $Z_{l}$ of section \#1 is split into 4 sections while $Z_{l}$ in sections \#2 \#5 are split into two sections to minimize parasitic shunt capacitors. Section \#6 represents the smallest realizable bandstop filter implemented in this design. For design simplicity, all sections have the same $Z_{c}$ and $c$ value of 61 Ohm and 0.26 , respectively. Their circuit parameters and frequency characteristic are summarized in the Table II.

Table II the simulated frequency characteristic of seven different unit element bandstop filters.

\begin{tabular}{|c|c|c|c|c|c|}
\hline$\#$ & $\begin{array}{c}f_{o} \\
(\mathbf{G H z})\end{array}$ & $\begin{array}{c}Z_{t} \\
(\Omega)\end{array}$ & $\begin{array}{c}\text { DC } \\
\text { Capacitance } \\
(\mathbf{p F})\end{array}$ & $\begin{array}{c}\text { Frequency } \\
\text { coverage } \\
(\mathbf{G H z})\end{array}$ & $\begin{array}{c}\text { Minimum } \\
\text { Stopband } \\
\text { attenua- } \\
\text { tion (dB) }\end{array}$ \\
\hline 1 & 4.88 & 7 & 4.9 & $2.5-8$ & 19 \\
\hline 2 & 10.6 & 10 & 2.03 & $4.5-15$ & 19 \\
\hline 3 & 17.2 & 17.2 & 0.71 & $11-20$ & 20 \\
\hline 4 & 28 & 11 & 0.53 & $16-34$ & 25 \\
\hline 5 & 35 & 10.2 & 0.39 & $23-47$ & 23 \\
\hline 6 & 40 & 23 & 0.3 & $28-57$ & 20 \\
\hline 7 & 45.5 & 40 & 0.11 & $44-47$ & 20 \\
\hline
\end{tabular}

Each element is strategically placed in series starting from small element to large element as it reaches the center of structure. This allows high frequency signal to be blocked by small elements with low radiation loss before it reached section \#1 and \#2 that have high radiation around the center of the filter. Short transmission line length with $Z_{0}=53 \mathrm{Ohm}$ is used to connect between element to minimize the filter total length. In addition, small input pocket is used around the filter terminal as shown in Fig. 4 to block low frequency radiation produced from the center of the structure,

\section{HARDW ARE IMPLEMENT ATION AND EXPERMMENT AL RESULTS}

The microstrip filter is fabricated on 0.127 mm-thick Rogers' Duriod 6010 substrate. It is attached to a copper enclosure using CW2400 conductive silver epoxy. The microstrip enclosure wall height is $2.54 \mathrm{~mm}$. The filter is connected to $2.4 \mathrm{~mm}$ connectors as shown in Fig. 5. The 
connectors' center coaxial pins are soldered to the wide microstrip pads at both ends of the filter.

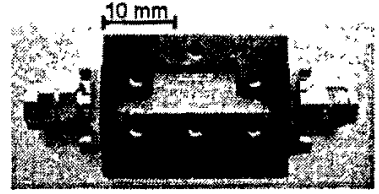

(a)

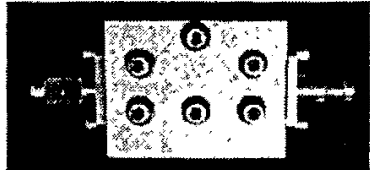

(b)
Fig. 5. The photograph of the microwave blocking filter in a package (a) with and (b) without top metal cover.

The filter is simulated using Ansoft Designer - method of moment simulator without the enclosure. It is also simulated using Ansof HFSS - finite element simulator - to study the effect of enclosure resonance cavity. The electro-magnetic (EM) simulation results are compared with measurement results using the Agilent PNA N5230A and produces good agreement as shown in Fig 6. The transmission line circuit model can predict the response well below $10 \mathrm{GHz}$ but cannot predict the stopband attenuation level at high frequency due to complex interaction among elements.

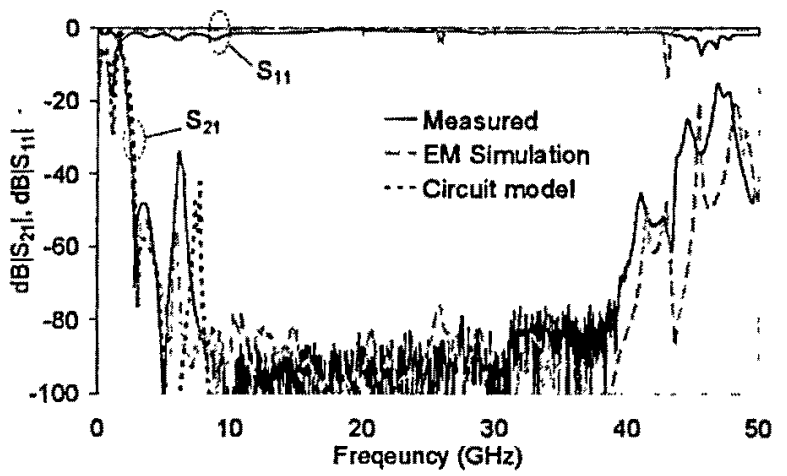

Fig. 6. The frequency responses of $\left|S_{21}\right|$ and $\left|S_{11}\right|$ of the measured and simulated microwave blocking filter.

From this result, one of the spurious responses at $6.3 \mathrm{GHz}$ is higher than that simulated. This is due to tolerance error in the coupled line spacing that changes transmission zero frequency locations in section $\$ 2$. Without the top metal cover that encloses the filter, the filter offers extended rejection bandwidth to more than $50 \mathrm{GHz}$ as shown in Fig 7. The effect of radiation can be observed above $40 \mathrm{GHz}$ when the filter is enclosed in a cavity. This is the result of the input pocket's length being too short as the frequency approaches the cavity's TE10 cut-off frequency. The measured capacitance and inductance excluding connectors is $22.5 \mathrm{pF}$ and $45 \mathrm{nH}$ at $10 \mathrm{kHz}$, respectively. The filter $\mathrm{DC}$ resistance is measured to be $0.925 \mathrm{Ohm}$ at room temperature.

\section{CONCLUSIONS}

The compact microstrip microwave blocking filter is proposed. Several techniques are used to increase filter stopband attenuation to more than $70 \mathrm{~dB}$ and extend stopband bandwidth up to the limit allowed by the fabrication process, for the first time. In addition, the exact solution of the transmission zero frequencies of the bandstop filter is derived.

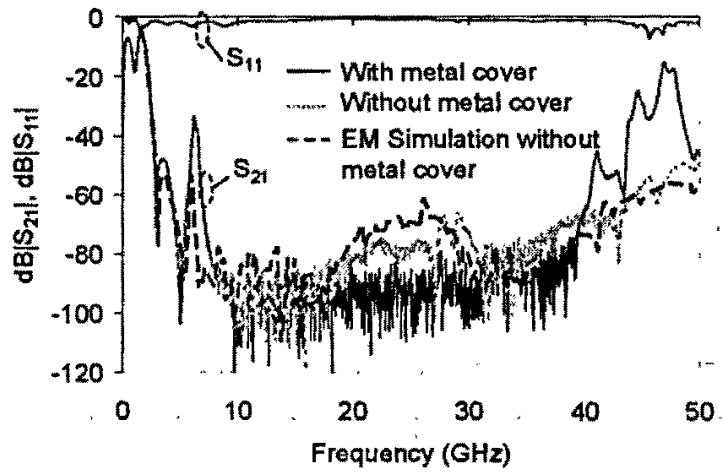

Fig. 7. The frequency response of $\left|S_{23}\right|$ and $\left|S_{n}\right|$ of the microwave filter with and without top metal cover

\section{ACKNOWLEDGMENT}

Author would like to thank Samuel H. Moseley and Nga T. Cao for providing measuring equipment and simulation support respectively.

\section{REFERENCES}

[1] K Bladh, D. Gunnarsson, E. Hurfeld, \$. Devi, C. Kristoffersson, B. Smslander, S. Pehrson, T. Claeson, and P. Delsing, "Comparison of cryogenic filters for use in single electronics experiments" ${ }^{*}$ Rev. SCi. Instrum, vol 74, no. 3, Mar 2003. pp. 1323-1327.

[2] A.B. Zorin, "The thermocoax cable as the microwave filter for single electron circuits," Rev. Sci. Instrum, vol. 66, no. 8, Aug 1995, pp. 4296-4300.

[3] A. Fukushima, A. Sato, A. Iwasa, Y. Nakamura, T. Komastsuzaki, and $\mathrm{Y}$. Sakamoto, "Altenuation of microwave filters for single-electron tunneing experiments," IEEE Trans. Instr. Measurement, vol. 46, no. 2 Apr 1997, pp. 289-292

[4] J. M. Martinis, M.H. Devoret, and J. Clarke "Experimental Tests for the Quantum Behavior of a Macroscopic Degree of Freedom: the Phase Difference across a Josephson Junction," Physical Review $B$, vol. 35, No. 10,1987, p. 4682

[5] P. Wahigren, "The Radio Frequency Single Electron Transistor and the Horizon Picture for Tumeling," PhD Thesis, Chalmers University of Technology, Gothenburg, Sweden, 1998, pp. 38-39.

[6] D. Vion, P.F, Orfila, P. Joyez, D. Esteve, and M.H. Devoret, "Miniahure electrical filters for single electron devices," J. Applied Physics, Vol. 77, No. 6, Mar 1995, pp.2519-2524.

[7] S. M. Lee, K. K. Ryu, I. B. Yom, S. P. Lee, "Novel low-pass filter for broad-band spurious suppression," in Proc. IEEE Int. Microwave Symp. Jun 2006, pp. 1797-1800

[8] L.H. Hsieh, K. Chang, "Compact, broad-stopband elliptic-function lowpass filters using microstrip stepped impedance hairpin resonators, ${ }^{\text {n }}$ in Proc. IEEE Int. Microwave Symp. Jun 2003, pp. 1775-1778.

[9] S. W. Ting, K W. Tam, R. P. Martins, MMiniaturizd microstrip lowpass filter with wide stopband using double equilateral U-shaped defected ground structure," IEEE Miorowave Wireless Comp. Let., vol. 16, no. 5, May 2006, pp, 240-242.

[10] M Y. Hich, S. M. Wang, "Compact and Wideband Microstrip Bandstop Filter," IEEE Microwave Wireless Comp. Let., vol. 15, no. 7 , Jul 2005, pp* 472-474.

[11] R. K. Hoffmann, Handbook of Microwove Integrated Curcuits, MA, USA: Artech House, 1987.

[12] D. M. Pozar, Microwave Engineermg, 2n ed., New York, USA: John Wiley \& Sons, 1998. 